\title{
Excitons in self-assembled quantum ring-like structures
}

\author{
H. Pettersson ${ }^{a}$, R. J. Warburton ${ }^{a}$, A. Lorke ${ }^{a}$, K. Karrai ${ }^{a}$, J. P. Kotthaus ${ }^{a}$, J. M. Garcia ${ }^{b}$ and \\ P. M. Petroff \\ a Center for NanoScience and Sektion Physik, Ludwig-Maximilians Universität, Geschwister- \\ Scholl-Platz 1, 80539 Munich, Germany \\ ${ }^{\mathrm{b}}$ QUEST and Materials Department, University of California, Santa Barbara, CA 93106, USA
}

\begin{abstract}
A remarkable morphological change of self-assembled InAs quantum dots takes place during growth if a pause is introduced after overgrowing the dots with a few $\mathrm{nm}$ of GaAs. Atomic force microscopy indicates that the shape of the dots changes lens-like to ring-like. We report here the results of capacitance and interband transmission experiments on such ring-like structures embedded in a GaAs matrix. In particular, we compare the electronic properties of conventional dots with those of the rings. Significant changes are found which qualitatively support a quantum ring model.
\end{abstract}

Self-assembly offers an attractive route for nano-structuring semiconductor materials. In particular, the Stranski-Krastanow growth mode can be used to produce InAs quantum dots in GaAs. The dots are typically lens-shaped [1]. However, this is not the only possibility. It has been shown that a pause in the growth after the deposition of the InAs dots and a few $\mathrm{nm}$ of GaAs induces a remarkable transformation in the dots' shape from lens-like to ring-like [2]. This is probably driven by lateral diffusion of indium. We report here experiments to probe the electronic structure of these rings.

The samples were all grown by solid-source molecular beam epitaxy. A nominal deposition of approximately 1.7 monolayers of InAs results in lens-shaped islands of $20 \mathrm{~nm}$ diameter and 6 $\mathrm{nm}$ height, with a typical density of about $10^{10} \mathrm{~cm}^{-2}$. If a short interruption of a few minutes is introduced after a $1 \mathrm{~nm}$ overgrowth of $\mathrm{GaAs}$, the shape of the quantum dots changes to a ringlike structure with an outer diameter of about $80 \mathrm{~nm}$ and an inner diameter of about $30 \mathrm{~nm}$ [2] (see inset in Fig. 1). The self-organized quantum rings are subsequently completely overgrown. The rings are embedded in a MISFET-type of heterostructure where a highly doped GaAs layer serves as a back contact, and a metal layer on the surface as a Schottky contact [3 and 4]. The distance between the GaAs back contact and the layer with rings is 
small enough to allow for tunneling which makes it possible to tune the electron occupancy of the rings.

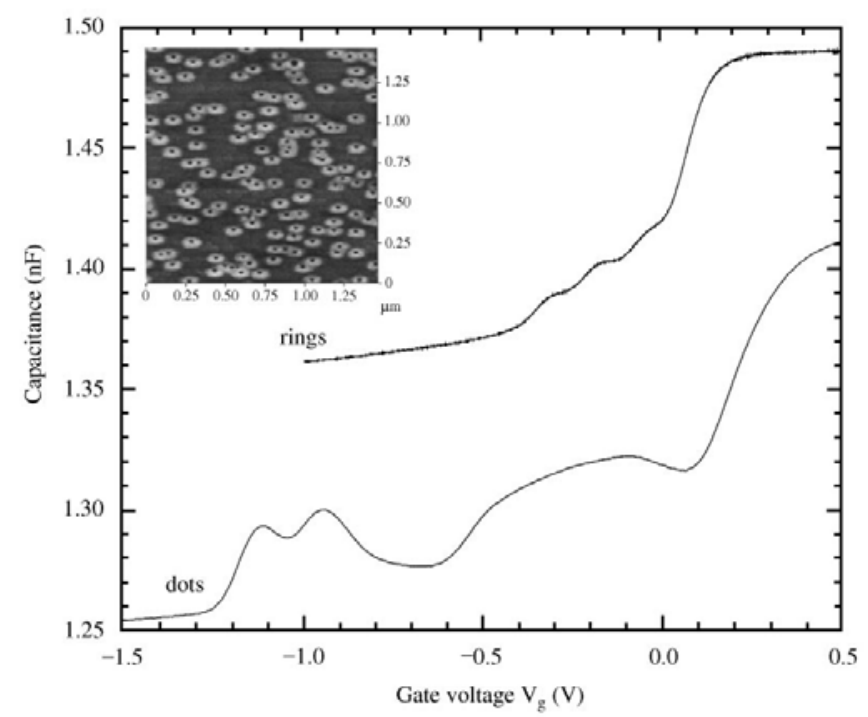

Fig. 1. The differential capacitance for a dot sample and a ring sample are plotted against gate voltage at $4.2 \mathrm{~K}$. In both cases the gate was circular with a diameter of $1.5 \mathrm{~mm}$. The curve for the rings is offset vertically for clarity. Inset shows an AFM image of the rings.

Fig. 1 shows typical capacitance traces obtained by measuring the differential capacitance between the Schottky contact and the buried back contact while sweeping the DC bias. The lower trace shows the differential capacitance spectrum of a dot sample. The observed resonances are due to tunneling of electrons from the back contact into discrete states of the dots. The twin peak at $-1 \mathrm{~V}$ and the broad peak at $-0.3 \mathrm{~V}$ correspond to tunneling into the twofold degenerate s-state and the four-fold degenerate excited p-state of a parabolic confinement. The splitting of the peak corresponding to tunneling into the s-state is due to the electron-electron interaction (Coulomb charging) and amounts to about $25 \mathrm{meV}$ [5]. At $0.1 \mathrm{~V}$, there is a rapid rise in the capacitance which corresponds to tunneling into the wetting layer [3 and 4]. The upper trace is a differential capacitance spectrum of a ring sample. Comparing the two traces we observe both similarities and differences. The features corresponding to tunneling into the rings are all shifted to much higher voltages but the rapid increase in signal corresponding to tunneling into the wetting layer is at the same voltage. The interpretation is that the rings have a higher ground state energy than the dots, pushing the charging peaks to higher voltage. This can be understood from the AFM measurements: the rings are thinner in the vertical direction than the dots, giving larger vertical confinement energies. The rings exhibit three charging peaks. By calculating the area under each peak, and taking into account the concentration of rings estimated from AFM measurements, we can deduce that each of the peaks corresponds to loading one electron into each ring. We thus conclude that the observed splitting of ca. $20 \mathrm{meV}$ between the first two peaks corresponds to the Coulomb charging energy for two ground state electrons in the ring. This energy is very similar to the charging 
energy of the dots despite the fact that the rings are considerably larger in diameter than the dots. This suggests that the rings have a different geometry than the dots. Furthermore, the three charging peaks for the rings are approximately equally spaced whereas there is a large gap between the second and third charging peaks for the dots. For the dots, this gap arises from the large quantization energy separating the s- and p-states. In other words, the dots are in the large quantization regime where the single-particle splittings are larger than the Coulomb interactions [6]. For the rings, the equidistant charging peaks are reminiscent of Coulomb blockade on lithographically defined dots which are in the weak confinement regime where the Coulomb energies dominate over the quantization energies. The implication is that in the rings there are states separated by energies considerably less than the charging energy of $20 \mathrm{meV}$. The large diameter of the rings implies that these states stem from rotational quantization.

In order to get more information on the electronic structure of the rings, we have performed transmission experiments in the near infrared to detect absorption through interband transitions. Such experiments have already been reported on InAs dots and are particularly useful here because they measure not only the energies but also the oscillator strengths of the various transitions [7]. The absorption measurements on the rings were performed with a Fourier transform spectrometer and a $\mathrm{Si} \mathrm{p}-\mathrm{i}-\mathrm{n}$ diode as detector. In Fig. 2 we show an absorption spectrum for dots together with an absorption spectrum for rings. In both cases, the gate voltage was more negative than the onset of electron tunneling so that we are measuring the properties of neutral rings and dots. Although the dot and ring densities were not identical, we have scaled the dot and ring absorption with the dot and ring densities, respectively, so that the intensities can be directly compared. For the dots we observe three transitions, which denote as $s-s, p-p$ and $d-d$, labeling the conduction and valence band states as $s, p$, and $d$ [6]. We observe also three peaks for the rings, but they have quite different properties.

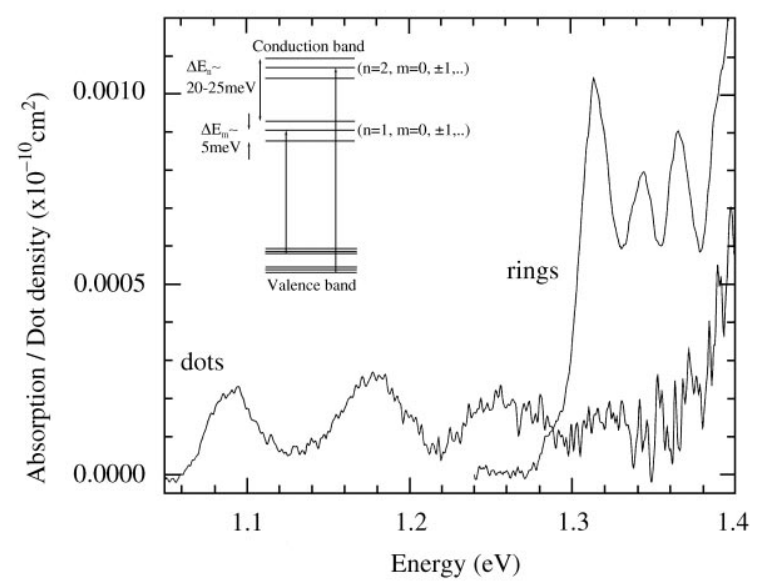

Fig. 2. Absorption per density of absorbers is plotted against photon energy for dots and rings at $4.2 \mathrm{~K}$. The absorption was determined by subtracting the measured transmission from 1 . The dot density and the ring density were determined from the capacitance measurements. Inset shows schematically the relevant electronic structure for the fundamental and the first excited transitions. 
We concentrate initially on the ground state transition. The most obvious effect is that the fundamental transition is at $1.31 \mathrm{eV}$ for the rings, yet only $1.1 \mathrm{eV}$ for the dots. This is consistent with the different onset of charging in the capacitance, and strongly supports the assertion that the vertical confinement energy is larger for the rings. Additionally, the oscillator strengths are quite different in the two cases. We integrate the area of the first charging peak in the capacitance traces to give the dot and ring densities. We then fit the absorption curves to four Gaussian curves, three for the dot or ring resonances and one for the absorption of the wetting layer at large energy. The area of each Gaussian is linearly related to the density of absorbers and the oscillator strength [6]. For the ground state transition, we find an oscillator strength $f=10.9$ for the dots, yet $f=31$ for the rings. The result for the dots can be understood in the strong confinement picture [6]. The oscillator strength represents simply the overlap between the electron and hole wave functions. The much increased oscillator strength for the rings implies that an exciton in a ring is in the weak confinement limit [8]. This is entirely consistent with the analysis of the capacitance spectra. In terms of the relevant length scales, the conclusion is that at least one dimension of the rings is larger than the excitonic Bohr radius. The picture is that the exciton moves as a composite particle in the complex potential landscape of the ring.

The excited interband transitions also emphasize the point that the rings are quite different from the dots. Firstly, the first excited transition has a lower oscillator strength (18) than the fundamental transition. Conversely, for the dots, the first excited transition is almost twice as intense as the fundamental transition simply because the degeneracy is a factor of two higher. Secondly, the first excited transition for the rings exhibits only a diamagnetic shift on applying a magnetic field, i.e. it has $\mathrm{s}$ character. For the dots, the first excited transition splits into two in magnetic field, i.e. it has $p$ character. Thirdly, we observe quite different behavior of the interband spectra on occupation with electrons.

It might be argued that all our data imply simply that the rings are dots with a smaller vertical dimension but a larger lateral extent. This would account for the shift to positive voltages of the charging peaks, the larger band gap, the equidistant charging peaks in the capacitance trace and the increased oscillator strength of the exciton. However, it is difficult to account for the large charging energy and in particular the existence of the excited interband transitions in this model. These transitions have the same orbital angular momentum as the ground state transition but yet have higher energy. We propose that the states of the ring can be labeled by two quantum numbers, $m$ and $n . m$ is the quantum number of angular momentum projected along the growth direction and $n$ is the quantum number describing the eigenstates in the radial confinement potential [9]. The separation of the states with different $m$ but the same $n$ can be estimated to be a few meV from the geometry of the rings. Conversely, we propose that the separation of states with the same $m$ but different $n$ is ca. $20 \mathrm{meV}$ for the electrons. In the interband transmission, we see transitions between different sets of radial states (see inset in Fig. 2). The transitions have an overall s character because various transitions with different $m$ are admixed by the Coulomb potential between electron and hole. These conclusions are in 
excellent agreement with far-infrared measurements of intraband transitions on these structures where a resonance was observed at $20 \mathrm{meV}$ [10] which can be attributed to a transition with $\Delta m= \pm 1$ and $\Delta n=1$.

In conclusion, we have studied self-organized InAs ring-like structures embedded in GaAs using capacitance and transmission spectroscopy. We have compared the results with those from conventional InAs dots and have found significant changes. Our experimental results are consistent, at least qualitatively, with the expected properties of a quantum ring for which the confinement in the radial direction is much larger than the confinement in the orbital direction.

\section{Acknowledgements}

The authors gratefully acknowledge fruitful discussions with A.O. Govorov and R.J. Luyken. One of the authors (H.P.) would like to thank the Alexander von Humboldt foundation for financial support and the group at LMU for their hospitality. This work is supported by the Deutsche Forschungsgemeinschaft (SFB 348).

\section{References}

1. D. Leonard, K. Pond and P.M. Petroff. Phys. Rev. B 50 (1994), p. 11687.

2. J.M. Garcia et al.. Appl. Phys. Lett. 71 (1997), p. 2014.

3. H. Drexler et al.. Phys. Rev. Lett. 73 (1997), p. 2252.

4. G. Medeiros-Ribeiros et al.. Phys. Rev. B 55 (1997), p. 1568.

5. B.T. Miller et al.. Phys. Rev. B 56 (1997), p. 6764.

6. R.J. Warburton et al.. Phys. Rev. B 58 (1998), p. 16221.

7. R.J. Warburton et al.. Phys. Rev. Lett. 79 (1997), p. 5282. 